\title{
結合現代物理学和其它方面的需要 发展泛函分析
}

\author{
关肇 直 \\ (中国科学院数学研究所)
}

泛函分析是研究无穷維空間和 它里面的分析学 (微积分学等)的数学分支。如果在 19 世紀唯心主义 哲学家对于四維空間已經作了不少荒謬的投机的話， 当然，談到无穷維空間，很容易給人以过于抽象，不 切实际之感。但作为一閏科学，它是从解决实际問題 的需要产生的。决定一个物理系統的状态的参数的个 数叫做这个采統的自由度。在稹点力学中，常遇到具 有穷自由度的系統。但在連續介质力学中, 往往遇到 具无穷自由度的力学系統 (例如振动的梁)。无穷維空 間正是反映具无穷自由度的系統的数学概念。

自从本世紀 20-30 年代，泛函分析开始作为一 椚新的独立学科形成以后，关于无穷維空閒的解析几 何学的研究已經发展得相当好了。首先是备內积空間, 其次是备賦范綫性空間，近年来对于賦可数范数的綫 性室間,乃至更一般的(肩部凸)拓扑綫性空間的研究， 已經积累了大量的成果，形成了泛函分析的分支。但 为了描写物理現像最为需要的无穷維空閒中的微积分 学，发展得还很不够，还有待于大力开拓。

很自然会想到, 在无穷維空間中取佰的实变量(表) 示时間) 函数乃是描写連續介盾的力学性盾的自然数 学工具 ${ }^{[1-2]}$ 。从另外一个角度看, 这种函数及可以表 达随机过程一一賦范綫性空間中的随机 元 $^{[4]}$ 。相应地, 这种期范綪性空間上的微分方程也就成为描写連續介 盾力学中的运动的工具。在很多实际問題中出現的随 机微分方程, 似乎也应当看作这种空間上的微分方程 而加以研究。实际上, 通过数理方程, 使泛函分析方 法在力学問題上发揮了作用。这种傾向从苏联 1956 年全苏联泛函分析会議, 1956 年全苏第三屆数学大会 和 1958 年泛函分析 会議上的报告 內容可以清楚地看

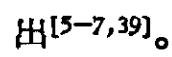

特別非绕性算子的研究是和为了更精确地反映客 覌現实而从螕性走向非螕性問題研究的总趋势分不开 的。如果說非綫性偏微分方程的数值解法是当前計算
数学中的核心問題，而非綫性偏微分方程的定性理諞 的建立又是微分方程論中的重要方向, 那末很自然地, 非綫性算子的研究在派函分析中也日益占着显要的位 置。值得注意，苏联学者們已把非綫性算子的理論和 方法用到各种实际問題上去。例如 M. A. 克拉斯諾 謝尔斯基把泛函分析方法用到非綫性振动問題上获得 了有意义的結果 ${ }^{[8]}$ 。还有反映众多的实际現象 (船的 运动，河流泛监，港口的水面等等)的表面波的研究， 也常化成非綫性算子的問題 ${ }^{[9] 。}$

广义函数的出現从根本上改变了函数概念。如果 定义在各点的函数只是一些物理量的近似描速的話, 那末，广义函数就成为描写很多物理現像的更自然的 工具。广义函数理論与数理方程論的結合近年来已既 为大覌, 抹在解决許多力学( 如气体动力学等) 問題上 发揮了作用 $[10,11]$ 。

由上述可見, 結合数理方程的研究, 針对力学中 各种实际問題, 开展泛医分析的許多方向的研究，乃 是具有辽閏的前途的，这里将不作更进一步的叙进， 这里地不去征引这方面待在的大量文献。

泛函分析更直接的作用是在微观世界的物理学方 面。早在本世紀三十年代,由于发現了物盾的二䅗性， 过去用来描述质点运动的和描述波現象的数学工具分 別使用当然不能适应这种二解的客覌实际, 而两套数 学工具又不能生硬地讲湊起来。把两套数学工具中的 互相抵触的性盾撤开, 而抽出它們的共同属性, 于是可 以看出, 用来表达物理量的不能再用 17 世紀以来一一直 使用的普通变量 (包括矢量), 而是要用无穷維內积空 間中的自伴綫性算子。这些物理量的可能观测值正是 相应算子的本征值，这正好适合了量子化的要求。于 是內积空間綫性自伴算子的譜理論适应着物理問題的 要求得到了进一步的发展, 在本世紀 30 年代达到了 成熟的地步。原子核的理論研究与这种表达方式是分 不开的。 
近年来基本粒子的研究是現代物理学中的中心問 題之一。为了了解基本粒子的相互作用，我們看作是 种种的場。这些場都是具有无穷自由度的，从而場方 程都是泛函方程。一方面, 由于这些場涉及微覌現象， 从而要量子化，于是物理量要用自伴綫性算子表示。 另一方面，由于用点型粒子表現事物只是一种极限看 法，師只是具有广表的事物的近似表迌，从而这种物 理規律应当表达成广义函数的方程。

从目前看来,現代物理学一一特別是原子核物理、 基本粒子理論一一所需要的数学表达方式由泛函分析 提供。但結合現代物理学开展泛函分析的研究，絕不 是仅仅指把泛函分析中的已有知識用到現代物理中 去, 而且, 由于目前量子場論方面还存在着巨大的困 难，其中一部分是由于还没有找到适当的数学工具， 因此, 很可能要創造新的数学理論和新的数学工具才 能完全解决这方面的問題。因此这方面的㸴究是大有 前途的。

下面只就目前已經遇到的一些問題, 提出来供大 家教孝。

首先，我們簡略地談一談量子場論。量子力学曾 被用来解决古典物理学所无法解决的許多問題，例如 原子結构、分子結构、固体結构和性稹等等, 从而量 子力学取得了重裂的成就。但从量子力学的运动方程 可以看出它不是相对論不变的，从而它不能用来处理 速度接近于光速的物理現缘。在粒子速度接近于光速 的現象中,粒子的动能可能大大超过它的静能,在粒子 相互作用的过程中能量轉換可能扩大，于是会产生新 粒子。例如用高能稹子和原子核相磁撞就产生核子和 反核子。量子力学只能描述数目一定的粒子的状态。 为此，在物理学中引进量子場的概念。量子場的各种 激发态代表处在各种状态中的粒子菜統。場的激发代 表粒子的产生，而激发的消灭代表粒子的消失。为了 描述不同种类的基本粒子，我們用不同的量子場。場 的总能量的不連續性（表达成相应的自件算子的譜是 散的)表达相应于这种場的粓于的存在 (例如电磁場 总能量的不連續性表明光的量子，郎光子的存在，等 等)。，客覌实际的場总是相互作用、相互联菜、相互 制約的。为了描述基本粒子的相互作用，要考虑不同 場之間的相互作用，这是量子場論中非常重要，也是 非常困难的工作。

为了解带相互作用的場的方程，很自然地引入散 射算子的概念。所䚴散射算子，乃是定义在由一切态 所形成的內积空間中的一个保范算子，它把始态变成 終态。这个算子在物理学中的重要性在于，它一方面
与所考察的过程的可覌測的特征有簡单的联系，另方面由自然界一些一般规律所导出的結論可以直接表 达成算子 $s$ 的一些性稹。例如算子 $S$ 的相 应陣 $\left(\omega_{a}, s \omega_{b}\right)$ 的絕对值平方就决定系統由态 $\omega_{b}$ 变到态 $\omega_{a}$ 的跃迁概率，也就是表达已知系統的力学量在开始状 态取某些值时在終結状态取种种值的概率，而各种守 唒定律就表达成相应算子和算子 $s$ 的可交換性一一包 括能量、动量、角动量、电荷以及宇称守恆。由散射 算子可以計算出两种类型的可覌測量，師䒺統的生存 时間和有效截面积。这里系統的生存时間是指系統在 单位时間丙变到一切可能态的跃迁概率的倒数, 而单 位时間粒子的跃迁概率与入射粒子流的比例常数叫做 有效截面积。

有了这些准备，問題就在于解場方程了。平常使 用迭代法来解，而与这个迭代程序相应，算子 $s$ 也展 成一个无穷級数

$$
s=\sum_{n=0}^{\infty} s^{(n)} .
$$

这級数中的每一項 $S^{(n)}$ 可以分解成很多項，每一項所 反映的物理作用乃是一些粒子的消失和一些粒子的产 生，也就是反映了某种粓子轉化的現象或某种粒子磁 撞的現象。

在对于具体物理現家进行計算时，如果只取最低 阶的近似，結果与实驗很符合。为了更精确，当然想 到献用更高阶近似，但事实上不但不能得到运好的近 似，反而得出发散的項来。这种发散的困难成为量子 場論发展的主要障碍。这是和場具有无穷自由度这一 事实密切相关的。

解决这一困难的一种方式乃是利用重新定义粒子 稹量的所謂盾量重正化的方法。在量于电动力学的場 合, 这样所得的結果与实驗完全相符, 成为量子場論 近年来最大成就之一。但重IE化方法捄不能解决量子 場論的一切发散困难。

一种想法乃是把困难归結到数学工具的不适当， 从而找寻合适的新数学表达方式也是消除困难的一利 尝試。

一种想法諳为古典分析經常使用的点函数实际_上 抹不能很好地反映現实，因为点粒子的表像只是- - 种 极限情形，只是有广装的事物所表現的現像的近似描 迅。因此; 用来陈逃量子場諭的应該是广义函数而不 是平常函数。于是物理规律表达成广义函数的方程。 既然这样，就要求能对广义函数作各种运算，如同对 平常函数那样。但如果广义函数在求微商等方淮㼭不 受限制，从而在应用上确比平常函数优越，那末：厂 
义函数却有一缺陷，垖扭非任意两个广义函数可以相 乘。实际上，借微扰論作散射陣时，就遇到表达因果 关系的因果函数的乘积，而这种因果圂数正是广义函 数，它在光錐 $x^{2}+y^{2}+z^{2}-c^{3} t^{2}=0$ 上有奇异性。 在形式上求因果函数乘积的动量表示时，就遇到在动 量的大值处发散的积分。这样，怎样給予这种发散积 分有穷值的問題便是很重要的了。苏联博哥留波夫学 派利用了所謂 $R$ - 运算定义了因果函数的乘积。具体 地說，利用证則化，問題化成被乘的广义函数的正則 逼近的乘积是否有弱极限的問題。首先証明了乘积作 为在基本空間 $C(a, r, n)$ 的一个子空間上存在，从而 下一步便是子空間上的泛函数延拓到整个空間上去的 問題。为了使延拓是确定的，所用的补充条件完全是 根据一般物理原理出发的，師要求 (a) 与經典理論相 对应; (b)保持态振幅的范数; (c)相对論不变性; (d)因 果性条件。于是溥哥留波夫等利用了 $R$-运算完成了上 迅的延拓。这样可以对量子場論中的发散积分給予有 穷值。这在量子电动力学中获得了很好的結果 ${ }^{[14-16]}$ 。 現代量子場論在用到介子核子相互作用时又遭到 困难。这时由于相互作用常数的值較大，不可能用微 扰論。于是有种种企图越出微扰論的限制。其中一种 企图乃是利用所謂色散关系方法。这仍是从散射算子 入手，但这时这个算子的性稹应当不借任何运动方程 来建立，而是从一般的物理要求出发。最早海森堡提 出的是相对諭不变性和保范性。但对于有些情形，在 研究散射陣結构时, 不只姴对实的能值去考察它, 还 要对于复的能僬考察，也就是要䃟究它的解析性稹。 为此，上批的物理要求就不够了。从物理上考虑应当 奥求滿足因果律。因果律的㴗求是指在类空曲面上不 同点的同时量測不可能互相影响, 不管这两点怎样近, 也就是表明任何信号不能按快于光速的速度传递。用 数学表示,也就是指两个算子在光錐之外是可交換的。 散射陣元的解析性稹和因果性的要求有密切关菜。而 一旦散射振幅的解析性建立起来，就江以朋复变量菡 数的定理导出一个积分关系。这关系吅是散射振幅所 应滿足的,叫做色散关系(名称是仿效古典电动力学的 形式上相似的公式而起的); 它表达了散射振幅的实、 㱆部分之間的关采。色散关菜在物理上所以重要，首 先在于它表达了直接能由实㯺覌測的量之閒的关系，

从而可以用实驗来检証。注意色敬关系是直接从一些 基本的物理假設用严謹的数学証明推导指来的，从而 对于色散关系的实驗检驗就为直接检証这些物理假定 提供了可能性。因此：不只理論物理学家，就是实驗 物理学家，也很重期色散关系。这些关系可以簡化实
驗分析。

为了証明色散关系，发生了下列数学問題:（1） 滿足某条件的广义函数是否容許在复数域內的解析延 拓? 而如果容許这种延拓，相应的解析性区域是怎样 的? 更确切地說, 这里的函数是形如 $f(k, x)$ 的, $k=$ $\left(k_{1}, \cdots, k_{m}\right), r=\left(x_{1}, \cdots, x_{n}\right), f$ 按变量 $x$ 是广义函 数, 按复变量 $k$ 是在某区域 $G$ 中的解析函数, 換句話 望，对于基本空關 $S$ 中的每个函数 $\boldsymbol{\varphi}$,

$$
\int f(k, x) \varphi(x) d x
$$

是 $G$ 中全純的函数。所謂广义函数 $f(x)$ 从实区域 $G_{0}$ 到复区域 $G$ 中的解析超拓, 是指具下列性质扞在 $G$ 中 为全純的函数 $f(x+i y)$ :

(1) 对于每个固定的实数 $y$, 函数 $f(x+i y)$ 对于 凡滿足 $x+i y \in G$ 的一切 $x$ 是按 $x$ 的广义国数;

(2) 对于每个 $p \in S$, 如果 $\varphi$ 的支杜含在 $G_{0}$ 中, 那末 $\int f(x+i y) \varphi(x) d x \rightarrow \int f(x) \varphi(x) d x, y \rightarrow 0, x+i y \in G$. 博哥留波夫等証明了一象关于广义圂数的解析超拓定 理。特別他証明了对 $\pi$-介子和核子的非前进的散射的 色散关系。这方面的工作还有待継續 $[13,17-19]$ 。

跳出微扰論，也就是跳出弱相互作用的限制的另 一企图乃是重新建立相互作用場的基本方程，抹求出 它們的准确解。这种新陈述的特点在于它們不是表达 算子間的关系，而是直接关于散射陣元的方程。这时 有必要本稹上扩充理論中的数学工具, 也就是引入泛 函(变分)微商和泛函(連續)积分。事实上，和量子力 学不同，在量子場論中，波函数应当被波泛函代替： 这是依賴于場函数 $u(\vec{x})$ 的泛函数, 而这个泛函数可以 表示成泛函积分。散射随元乃是这个泛函数的变分微 商。利用这种泛函的陈迅来解决量子物理学中的問題 还是开始不久的, 因此相应的数学工具还有待于深入 研究。目前，量子場諭中要用到的連就积分对于什么 样的泛函数和在什么样的函数空間上能用有穷維积分 取极限来定义，也还是没有完全解决的问題。这方面 的問題已可起我国学者的注意。結合物理問題的实际 需姴，研究連續积分的定义。方式和計算閔題都是很储 得注意的 ${ }^{[20,22]}$ 。

非自件算子和特征陣函数的理碖可以用来研究在 基本粒子或原子核酳撞时所形成的中間系統 ${ }^{[23]}$ 。在很 多重䨋情形(例如共振散射、光被自由电子散射等), 由 某柔統的始态 $X$ 到終态 $Y$ 的跃迁不是直接发生的，而 是按照图式 $X \rightarrow C \rightarrow Y$, 这里 $C$ 是中間系統, 这个系粒 先产生 $(X \rightarrow C)$, 然后又消灭 $(C \rightarrow Y)$ 。我們要孝虑 $C$ 的 生存时間。系統 $C$ 的性稹本稹上决定了过程 $X \rightarrow Y$ 。由 $\mathrm{X}$ 到 $\mathrm{Y}$ 的轉化用散射陣 $S$ 描述: $Y=S X$, 而由 $S$ 可以 
找出一切可覌測量。找出 $s$ 与中間䒺統的关联是很有 意义的，特別要考察系統 $C$ 是否由 $S$-陣唯一决定。 由一般的假定出发, 可以作非稳定系統 $C$ 的能量 (非 自伴)算子 $A$ ，抹写出它的蛻变方程。这时 $S$ - 随等同 于算子 $A$ 的特征函数。因此借特征陣函数来分析非自 俄絊性算子，正是用散射随来分析中間系統。

內积室間中綫性算子的理論还有助于按照新的严 格性标准建立散射的理諭。这种用算子理論重新陈逝 物理散射过程的尝試也还有待于継續 ${ }^{[26]}$ 。

量于場諭中許多問題引出具不定尺度的內积空 間。这种空間中的量子場論成为不少学者注意的中 心 ${ }^{[32]}$ 。另一方面, 苏联数学家也已建立了一套具不定 尺度的空間中的算子理諭。把这两方面的工作結合， 仍是值得努力的 ${ }^{[41]}$ 。

由散射随反过来决定能量算子的問題，垖所謂反 問題具有重大实际意义，因为这問題的解决有助于說 明散射时的实驗数据，推决定粒子的不能直接由实驗 量測的一些特征。这对于了解原子核內部情况很有用 处。这方面的結果也还沟有完全解决 ${ }^{[24-25]}$ 。

还有不少努力，想借內积空閒的无穷积来描迅具

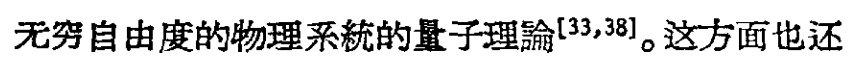
没有足够的成功。

如果可分备內积空閒中的自伴綫性算子理論給量 子力学提供了有力的工具, 扗在这方面的要求下得到 更大的发展，那末，在今天看来有关內积空間的上迅 一些問題的研究仍将对解决原子核和基本䊀子理論中 的䚺多問題起重要作用。

綜合上迅，非綫性算子理論，广义函数理論，无 穷維空間中的微积分学，內积空間中的自伴和非自体 䋐性算子的譜理論，在无穷維空間中取值的圂数的理 諭等, 就成为結合現代物理学和其他实际需要应当着 重发展的泛函分析分支。当然，这些分支又将带动各 种空間的研究。但我們䚯为, 这些研究仍应当針对上 面提到的实际問題的需要, 避宏作单純的推广。推广 性的工作也汼非不可以考虑。但这方面似乎也应考虑 “現代化”。換何話說, 利用現代数理遂輯的技巧, 解 决泛函分析中一系列制定問題，制定泛囦分析中哪些 类定理在某种場合真也就必然在另外的，更一般的場 合真。这就是泛函分析証明論的方向。这方面的成功 将彻底結束那些个別推广的工作。

以上都是很粗线的看法。現在全国很多地方，讪 函分析的研究已都有些基础。希望各地根据各种不同 方面的实际需要，結合原有基础，确定自己的方向。 各地方的工作没有必要雷同。同时，在全国各地协作
配合之下，在理論联系实际的方針之下，全国泛函分 析工作及是比較全面的。希望通过大家的討論，明确 方向，在党的領导下，鼓足干劲，力爭上游，相信在 不长的几年內，一定可以在运用泛函分析的知識和方 法解决現代物理学和其它各方面的实际問題上攀世 界高峯!

\section{考参 文 献}

这里的目录并浔有企图作成完备, 只是开列一批交策, 传 得通过这些交竟对所提的問題获得概括性的了解, 也可以通 过它們得到更多的参考文献。

[ 1 ] Pallioux, H. Quelques applications du calcul fonctionnel à la mécanique rationnel, Ann. Sc. Ec· Norm. Sup. 69 (1952), 213-257.

[2] Pailloux, H. Nonvelles applications du calcul fonctionnel à la mécanique, Ann. Sc. Fc. Norm. Sup. 70 (1953), 1-49.

[3] Hopf, E. On the application of functional calculus to the statistical theory of turbulence, Proc. Seventh Symposium appl. math. Amer. Math. Soc. 1955, vol. 7.

[4] E. Mourier et R. Fortet: Lesfonctions aléatoires comme éléments aléatoires dans des espaces de Banach. Studia math. 15 (1956), 62-79, 又 J. math. p. appl. 38 (1959), 347-364. 这方面此外还 有許多文献。

[5] Усп. Матем. Наук, 11:6 (1956), 12:1 (1957).

[6] Труды Третьего Bсесоюзного Математического Сьезда, Том I, II, III, IV.

[7] Межвузовская Қонференшия по функциональному аналязу я его прнменениям, УМН 14:3 (1959), 221-226.

[8] М. А. Красносельскпй О применении методов функционального анализа к задачам о нелинейных колебаниях, Труды Третьего Всесоюз. Мат. Съезда, Том III, 261-268.

[9] Теория поверхностных волн (Красносельский 圭編 1959)。

[10] Sauer, R. Einführung in den Kolkül der Distributionstheorie mit Anwendungen auf Anfangswertprobleme in der Gas-dynamik, Abh. Math. Sem. Univ. Hamburg 22 (1958), 50-70.

[11] K. R. Darfner. Dreidimensionale Überschallprobleme der Gasdynamik, 1957.

[12] Н. Н. Боголюбов п Д. В. Ширков. Введенне в теорню квантованных полей, 1957.

[13] Н. Н. Боголюбов, Б. В. Медіедев и М. К. Поливанов Вопросы теории дисперсионных соотношений, 1958. (注意这本书的后附交献)。

[14] Н. Н. Боголюбов-О. С. Парасюк. О вычитательном формализм при умножении иричинных Функций, ИАН сер. матем. 20 (1956), 585610.

[15] Парасюк, О. С. Умножение прицинных фуяк- 
ций при несовпадающих аргументах, ИАН сер. матем. 20 (1956), 843-852.

[16] Боголюбов, Н. Н. П Парасюк, О. Т. Über die Multiplikation der Kausalfunktionen in der Quantentheorie der Felder, Acta Math. 97 (1957), $227-266$.

[17] Н. Н. Боголюбов-В. С. Владимиров. Об аналчтическом шродолжении обобщенных функций, ИАН. сер. матем. 22 (1958), 15-48.

[18] В. С. Владимиров, Об определения области аналитичности, ИАН. сер. матеи. 23 (1959), $275-294$.

[19] В. С. Владимиров-А. А. Логунов. Об аналитических свойствах обобшеннюх функций квачтовой теорип поля, ИАН. сер. матем. 23 (1959), $661-667$.

[20] И. М. Гельфанд п А. М. Яглом. Интегрирование в функциональных пространствах и его применения в квантовой фпзике, УМН 11:1 (1056), $77-114$.

[21] И. М. Гельфанд. О некоторых проблемах функционального анализа, УМН 11:6 (1956), 3-12.

[22] Проблемы современной физика, 1955, 3, квантовая теория поля, 1955.

[23] М. С. Бродский-М. С. Лившиц. Спектральный анализ несамосопряженных операторов и промежуточные системы, УМН 13:1 (1958), 3-85.

[24] Л. Д. Фаддеев, Обратная задача квантовой теория рассеяния, УМН 14:4 (1959), 57-119.

[25] В. К. Мельников. О приближенных методов в обратной задаче кванговой теория рассеяния, УMH 14:4 (1959), 121-131.

[26] J. M. Jauch. Theory of the scattering operator, Helv. phys. acta 31 (1958), 127-158, II, 661684.

[27] J. Lehmer-G. M. Wing. On the spectrum of an unsymmetric operator arising in the transport theory of neutrons, Comm. p. appl. math. 8 (1955), 217-234.

[28] Goto, Ken-iti. On a regular formulation of quan- fum field theory, Progr. th. phys. 15 (1956), $176-177$.

[29] Iwata, Giiti. A formulation of field theory in Hilbert space, Progr. th. phys. 11 (1954), 537556.

[30] Fano, O. Description of states in quantum mechanics by density matrix and opeator techniques, Rev. mod. physics, 29 (1957), 74-93.

[31] Schmidt, W.-Baumann, K. Quantentheorie der Felder als Distributionstheorie, Nuovo Cimento, 4 (1956), 860-886.

[32] Нелинейная квантовая теория поля, 1959.

[33] S. Albertoni-F. Duimio. Hilbert spaces in quantum field theories, Nuovo Cimento, 6:5 (1957), 11931205.

[34] K. O. Friedrichs. Mathematical Aspects of the quantum theory of fields, 1953.

[35] Costa de Beauregard, O. Thérie synthétique de la relativité restreinte et des quanta, 1957.

[36] Матвеев, А. Н. Oџерационный метод в квановой теорип поля, Вест. МГУ 1953:10, 99-104.

[37] Deprit, A. Algèbre symétrique et seconde quantification d'un système de bosons, Ann. Soc. Sc. Bruxelles, 68 (1954), 23-33.

[38] I. E. Segal. Foundations of the theoy of dynamical systems of infinitely many degrees of freedom, I, Mat. fys. Medd. Kong. Danske Vidensk. Selskab, Bd 31, No. 12 (1959), 1-38.

[39] М. А. Красносельский, М. А. Наймарк и Г. Е. Шилов: Функциональный анализ, Математвка в СССР за сорок лет 1917-1957, 675-779.

[40] Проблемы современной физики, 1957:2, дисперсионные соотношения.

[41] Парасюк, О. С. и др. Теорема Челлена-лемана в пространстве с йндефянитной метрике, УК. МЖ. 10 (1958), 344-346.

[42] Иохвндов, И. С. п Крейн, М. Г. Слектральная геория операторов в пространствах с индефанитной метрикой, 1, Тр. Моск. Мат. Об-ва, 5 (1956), 367-432, II, 8 (1959), 413-496. 